\title{
Correlated Light Scattering by a Dense Distribution of Condensation Droplets on a Window Pane
}

James A. Lock

Cleveland State University, j.lock@csuohio.edu

Chin-Lien Chiu

Follow this and additional works at: https://engagedscholarship.csuohio.edu/sciphysics_facpub

Part of the Physics Commons

How does access to this work benefit you? Let us know!

\section{Publisher's Statement}

This paper was published in Applied Optics and is made available as an electronic reprint with the permission of OSA. The paper can be found at the following URL on the OSA website: http://www.opticsinfobase.org/ao/abstract.cfm?URI=ao-33-21-4663. Systematic or multiple reproduction or distribution to multiple locations via electronic or other means is prohibited and is subject to penalties under law.

\section{Original Citation}

Lock, James A. and Chin-Lien Chiu. "Correlated Light Scattering by a Dense Distribution of Condensation Droplets on a Window Pane." Applied Optics 33 (1994): 4663-4671.

\section{Repository Citation}

Lock, James A. and Chiu, Chin-Lien, "Correlated Light Scattering by a Dense Distribution of Condensation Droplets on a Window Pane" (1994). Physics Faculty Publications. 56.

https://engagedscholarship.csuohio.edu/sciphysics_facpub/56

This Article is brought to you for free and open access by the Physics Department at EngagedScholarship@CSU. It has been accepted for inclusion in Physics Faculty Publications by an authorized administrator of EngagedScholarship@CSU. For more information, please contact library.es@csuohio.edu. 


\title{
Correlated light scattering by a dense distribution of condensation droplets on a window pane
}

\author{
James A. Lock and Chin-Lien Chiu
}

\begin{abstract}
An analytical model of the scattering structure factor for an assembly of noninteracting hard disks has recently appeared in the literature [Phys. Rev. A 42, 5978-5989 (1990)]. We employ this model to calculate correlated light scattering by monodispersions and binary mixtures of condensation droplets on a window pane. We find that an area fraction of $f \geq 0.6$ is required for producing the near-forward direction scattering suppression and that a moderately wide polydispersion of droplet sizes is capable of producing the experimentally observed bright ring of colored light.
\end{abstract}

\section{Introduction and Overview of Correlated Scattering}

If one looks at a distant street lamp through a window pane fogged by condensation, one often sees a bright ring of colored light surrounding the street lamp. 1 The ring of light is red at its outer edge and blue at its inner edge. The region between the inner edge of the ring and the street lamp is dark (see Plate 44). Under especially favorable circumstances one sometimes sees a second dim ring of light surrounding the bright main ring. The qualitative explanation of this phenomenon has long been known. ${ }^{2-4}$ The distant street lamp acts as a coherent light source over distances of many condensation droplet diameters. Light diffracted by the perimeter of each of the condensation droplets reaches the observer. This light possesses a phase that depends on the positions of both the droplet on the window pane and the observer. The diffraction pattern is modulated by the interference of the phases of the light scattered by all the droplets. The destructive interference of the phases for near-forward scattering causes the dark region immediately around the street lamp, and constructive interference causes the bright ring of colored light surrounding the dark region. Cloud corona is also the result of diffraction of sunlight or moonlight by small water droplets in clouds. ${ }^{4,5}$ The reason why the center of the cloud corona pattern is

J. A. Lock is with the Department of Physics, Cleveland State University, Cleveland, Ohio 44115 . C-L. Chiu is with the Department of Computer Science, Pennsylvania State University at Shippensburg, Shippensburg, Pennsylvania 17257.

Received 9 September 1993; revised manuscript received 12 November 1993.

0003-6935/94/214663-09\$06.00/0.

(1) 1994 Optical Society of America. bright rather than dark is because water droplets are separated by many diameters in a cloud whereas they are nearly touching on a window pane. This difference in density greatly influences the interference modulation of the diffraction pattern.

As the interference of the phases of the diffracted light from the condensation droplets plays a major role in producing the dominant features of scattering by a fogged window pane, this phenomenon is an example of what is known as correlated scattering. ${ }^{6}$ The intensity of the bright ring is greater than that caused by incoherent scattering by a purely random distribution of particles, in which the intensities that are due to individual particles are additive. Yet it is less than that caused by coherent Bragg scattering by a crystal, in which each particle is located at a well-defined lattice position. Because the condensation droplets on a window pane are nearly touching, they are analogous to a two-dimensional liquid. If the center of a given droplet of radius $a$ is at the coordinate $\mathbf{r}_{i}$ and the center of another droplet is at $\mathbf{r}_{j}$, geometric packing constraints alone dictate that the nearest neighbors and next-nearest neighbors are preferred distances away from the given droplet, even though, over large distances, the relative positions of the droplets are random. The geometry of the scatterers is then one of short-range semiorder and long-range disorder. This produces scattering that is stronger than incoherent scattering by randomly positioned particles yet weaker than coherent scattering by particles on a crystal lattice.

There are a number of mathematical models of correlated scattering. In general, the more sophisticated the model, the more predictive power it has. In perhaps the lowest-level model, the condensation droplets on the window pane are considered as form- 
ing many domains of a polycrystalline array. ${ }^{7-9}$ If light is normally incident, the aggregate of the Bragg spots that are due to each domain forms circular rings, the innermost of which occurs at the scattering angle $\theta$, which is given by

$$
d \sin \theta=\lambda,
$$

where $\lambda$ is the wavelength of the light, and $d$ is the nearest-neighbor separation. The intensity of the scattered light at the Bragg angles is proportional to the scattered intensity of a single particle of radius $a$. If the random nature of the droplet positions is modeled by a perturbation of the location of each condensation droplet by a small random amount, the first Bragg ring becomes broader and less intense while the others are extinguished, qualitatively reproducing the fogged window-pane observations. We call this model the two-size model (i.e., droplet radius and separation) of correlated scattering. The greatest defect of this model is that, although it is successful in predicting where the bright scattered ring will occur and why the center region is dark, it cannot quantitatively predict the exact behavior of the scattered intensity as a function of angle. It also cannot trace the evolution of features in the scattered intensity as a function of droplet density from the dilute cloud corona limit to the high-density window-pane limit.

A brute-force method for calculating correlated scattering consists of measuring the positions $\mathbf{r}_{i}$ of all the droplets and performing the scattering sum

$$
\mathbf{E}(\theta)=\sum_{i=1}^{N} \mathbf{E}_{a}(\theta) \exp \left[i\left(\mathbf{k}_{0}-\mathbf{k}_{s}\right) \cdot \mathbf{r}_{i}\right]
$$

directly, where $\mathbf{E}_{\alpha}(\theta)$ is the scattered electric field that is due to a single particle of radius $a, \mathbf{k}_{0}$ is the wave vector of the incident light (see Fig. 1), and $\mathbf{k}_{s}$ is the wave vector of the scattered light. The complex

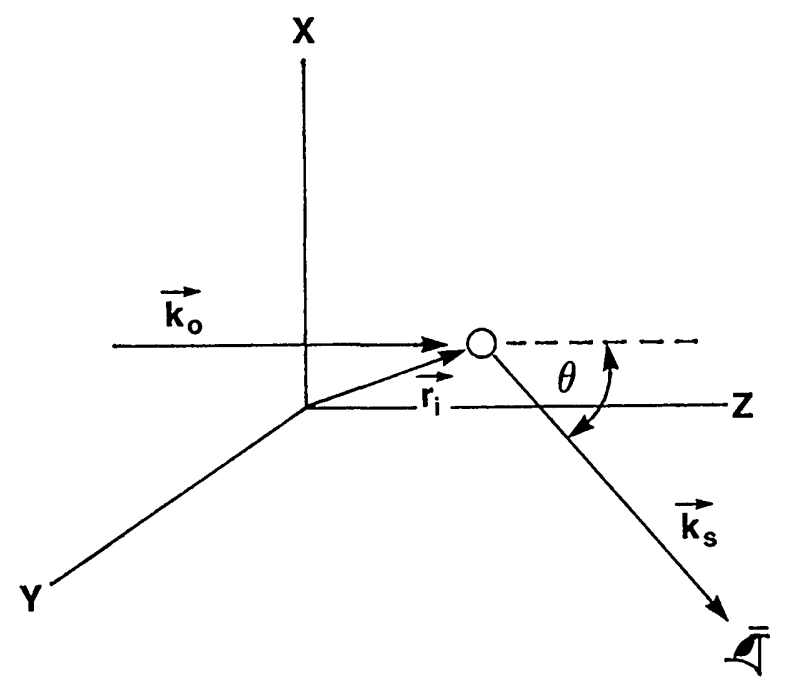

Fig. 1. Geometry for correlated scattering. The $i$ th scatterer is located at $\mathbf{r}_{i}$, the incident wave vector is $\mathbf{k}_{0}$, and the scattered wave vector is $\mathbf{k}_{s}$. The scattering angle is $\theta$. exponential in Eq. (2) is the position-dependent phase of the light scattered by the $i$ th particle. The direct summation procedure gives reasonable results if the experimental scatterer positions $\mathbf{r}_{i}$ are accurately measured..$^{10}$ But it may produce a few prominent streaklike scattering artifacts in simulations if dot scatterers are painted by hand onto a transparent substrate and subtle artist-generated patterns are present in the purportedly random distribution. ${ }^{4}$

A more sophisticated mathematical model for calculating correlated scattering employs the static paircorrelation function ${ }^{11} g(\mathbf{r})$. If $\sigma\left(\mathbf{r}_{j}\right)$ is the average macroscopic number density of condensation droplets at the position $\mathbf{r}_{j}$ and if $\Delta\left(\mathbf{r}_{j}-\mathbf{r}_{i}\right)$ is the local microscopic number density of droplets at $\mathbf{r}_{j}$, given that there is a droplet at the nearby position $\mathbf{r}_{i}$, the static pair-correlation function $g(\mathbf{r})$ is defined by '

$$
\Delta\left(\mathbf{r}_{j}-\mathbf{r}_{i}\right)=g\left(\mathbf{r}_{j}-\mathbf{r}_{i}\right) \boldsymbol{\sigma}\left(\mathbf{r}_{j}\right) .
$$

It is interpreted as the position-dependent fluctuation in the local microscopic number density from the average macroscopic number density. For an isotropic material, $g(\mathbf{r})$ depends only on the magnitude of $\mathbf{r}$. At the nearest-neighbor and next-nearest-neighbor positions, where the local microscopic density is larger than average, $g(r)>1$, and between the nearest-neighbor and next-nearest-neighbor positions, where the local microscopic density is smaller than average, $g(r)<1$. As the droplets cannot overlap, $g(r)=0$ for $r<2 a$, and, as the arrangement of the droplets is disordered when examined over large distances, $\lim _{r \rightarrow \infty} g(r)=1$. The dependence of the pair-correlation function on the density of droplets is illustrated in Fig. 2.

If the positions of the droplets are experimentally known, a histogram representation of $g(r)$ may be computed by statistical methods. ${ }^{11}$ Alternatively, in simulations random nonoverlapping scatterer positions are generated by a computer, and a histogram of $g(r)$ is again computed by statistical methods. This method has been employed extensively, ${ }^{12-15}$ because the two-dimensional noninteracting hard-disk system (i.e., the generic system for which condensation droplets on a window pane is an example) possesses a long-range order-disorder phase transition at an area fraction $f \approx 0.69$, where

$$
f=\pi a^{2} \sigma
$$

is the fraction of the total area covered by disks. Because $g(r)$ depends on the area fraction and because the scattered intensity depends on $g(r)$, the intensity exhibits different features as $f$ is varied.

A third method of computing the pair-correlation function is by the use of an analytical model of disk packing. A recently employed example is the freeenergy model.16 In this model the Fourier transform of $g(r)$ for the noninteracting hard-disk system is obtained as an analytical function. As is shown in Section 2, the scattered intensity is proportional to the Fourier transform of the pair-correlation function. 


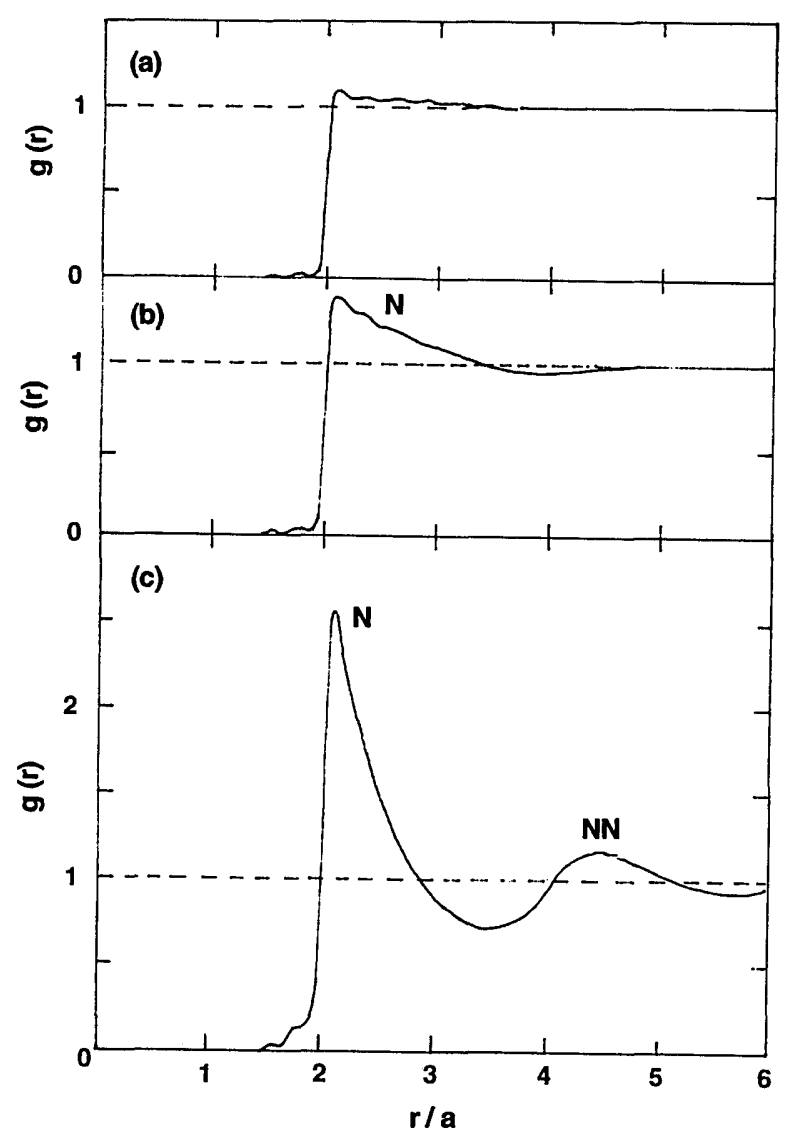

Fig. 2. Pair-correlation function for noninteracting hard disks with area fractions (a) $f=0.05,(\mathrm{~b}) f=0.2$, (c) $f=0.5$ employing the free-energy model of Section 2. The nearest-neighbor peak is denoted by $\mathrm{N}$ and the next-nearest-neighbor peak is denoted by NN.

Thus scattering calculations that use a histogram representation of $g(r)$ are prone to numerical convergence problems. These problems are bypassed if an analytical approximation to the Fourier transform of $g(r)$ is employed.

The purpose of this paper is to examine the details of correlated scattering in the free-energy analytical model for the Fourier transform of $g(r)$. The paircorrelation-function model of scattering by droplets on a window pane merits study, as it provides many insights into the scattering process that the two-size model cannot. In particular, it handles in a natural way the situation of condensation droplets of many different sizes and the effect this has on the scattered intensity. The body of this paper proceeds as follows. In Section 2 we briefly summarize the pair-correlationfunction model of correlated scattering by monodisperse particles and by particles of two different sizes. We also give the free-energy model analytical formula for the Fourier transform of $g(r)$. In Section 3 we present the results of our scattering calculations. Finally in Section 4 we analyze photographs of correlated scattering by condensation haze on a camera lens in order to determine the average size of the haze particles.

\section{Pair-Correlation-Function Model of Correlated Scattering}

Consider $N$ condensation droplets of radius $a$ on a window pane at the random positions $\mathbf{r}_{i}$ for $1 \leq i \leq$ $N$. Let light of wavelength $\lambda$ and wave vector $\mathbf{k}_{0}$ be normally incident upon the window pane, as in Fig. 1. The scattered electric field is given by Eq. (2), and the scattered intensity may be written as ${ }^{11}$

$$
\begin{aligned}
I(\theta)= & \mathbf{E}^{*}(\theta) \cdot \mathbf{E}(\theta)=I_{a}(\theta) \\
& \times\left\{N+\sum_{i=1}^{N} \sum_{\substack{j=1 \\
j \neq i}}^{N} \exp \left[i \mathbf{K} \cdot\left(\mathbf{r}_{j}-\mathbf{r}_{i}\right)\right]\right\},
\end{aligned}
$$

where $I_{a}(\theta)$ is the scattered intensity that is due to a single droplet of radius $a$. The quantity

$$
\mathbf{K} \equiv \mathbf{k}_{\mathbf{0}}-\mathbf{k}_{\boldsymbol{s}}
$$

is known as the scattering momentum transfer. For elastic scattering, as we have in the window-pane situation,

$$
|\mathbf{K}|=K=2 k \sin (\theta / 2) .
$$

The first term in Eq. (5) is the incoherent scattering sum, and the second term represents the interference of the phases of light scattered by all the droplets. If the sums over droplets are converted to integrals over the window-pane coordinates, Eq. (5) becomes ${ }^{11}$

$$
\begin{aligned}
I(\theta)= & I_{a}(\theta)\left\{N+N \sigma \int \mathrm{d}^{2} r[g(\mathbf{r})-1] \exp (i \mathbf{K} \cdot \mathbf{r})\right. \\
& \left.+\sigma^{2}\left|\int \mathrm{d}^{2} r \exp (i \mathbf{K} \cdot \mathbf{r})\right|^{2}\right\},
\end{aligned}
$$

where we have assumed that the macroscopic number density of condensation droplets is constant over the window pane. As in Eq. (5), the first term in Eq. (8) represents incoherent scattering, and the second term represents the scatterer phase correlation. The third term represents diffraction by the entire collection of scatterers. It ensures that scattering in the forward direction is coherent. But it is negligible compared with the first two terms when $\theta$ is greater than a few thousandths of a degree. As its importance does not extend beyond the observed angular size of the source, the third term in Eq. (8) is omitted in the remainder of this work.

For an isotropic system, the azimuthal integral in Eq. (8) may be performed to give

$$
\begin{aligned}
I(\theta)= & N I_{a}(\theta)\left\{1+2 \pi \sigma \int_{0}^{\infty} r \mathrm{~d} r[g(r)-1]\right. \\
& \left.\times J_{0}(2 k r \sin \theta)\right\}=N I_{a}(\theta) S(\theta),
\end{aligned}
$$

where the quantity in braces is called the scattering structure factor, and where $J_{0}$ is the zero-order Bessel function. With the exception of the leading factor of unity and the number density $\sigma$, the structure factor 
is the Fourier transform of $g(r)-1$. If scattering by each condensation droplet is dominated by diffraction, then

$$
I_{a}(\theta)=\frac{\pi^{2} a^{4}}{\lambda^{2} R^{2}} I_{0}\left[\frac{2 J_{1}(k a \sin \theta)}{k a \sin \theta}\right]^{2},
$$

where $I_{0}$ is the intensity of the incident light, and $R$ is the distance from the window pane to the observer. In actuality, $I_{a}(\theta)$ for near-forward scattering should also contain the effects of specular reflection off the droplet and transmission through it. ${ }^{5}$ But because these two effects are proportional to $a^{2}$ and because the observed scattering pattern corresponds to scattering angles smaller than that of the first diffraction minimum at

$$
\sin \theta \approx \frac{1.22 \lambda}{2 a}
$$

Eq. (10) is a reasonable approximation to $I_{a}(\theta)$ in the angular region of interest. From Eq. (9) one can see why $g(r)$ in histogram form obtained from statistical data may cause numerical convergence problems when the integral over $r$ is performed. As $r$ approaches infinity, $g(r)-1$ approaches zero while $r J_{0}$ $(2 k r \sin \theta)$ diverges as $r^{1 / 2}$. If $g(r)-1$ has not been computed for sufficiently large $r$ or if the statistical noise in the histogram is sufficiently large, the $g(r)-$ 1 factor will not counterbalance the divergence of the $r J_{0}(2 k r \sin \theta)$ factor. Employing an analytical model for $S(\theta)$ sidesteps these convergence considerations.

Thus far it was assumed that all the condensation droplets on the window pane were the same size. In order to model the effects of scattering by a polydispersion of condensation droplets crudely we now examine scattering by particles of two sizes. Consider $N_{1}$ droplets of radius $a_{1}$ and $N_{2}$ droplets of radius $a_{2}$. Their average macroscopic number densities are $\sigma_{1}$ and $\sigma_{2}$, respectively, and their area fractions are

$$
\begin{aligned}
& f_{1}=\pi a_{1}^{2} \sigma_{1}, \\
& f_{2}=\pi a_{2}^{2} \sigma_{2} .
\end{aligned}
$$

The total area fraction is

$$
f=f_{1}+f_{2} .
$$

Often the individual area fractions $f_{1}$ and $f_{2}$ are desired if one knows only the total area fraction $f$ and the indivdual number densities. The individual area fractions are then given by

$$
\begin{aligned}
& f_{1}=\frac{f}{1+\left(\frac{a_{2}}{a_{1}}\right)^{2} \frac{\sigma_{2}}{\sigma_{1}}}, \\
& f_{2}=\frac{\left(\frac{a_{2}}{a_{1}}\right)^{2} \frac{\sigma_{2}}{\sigma_{1}} f}{1+\left(\frac{a_{2}}{a_{1}}\right)^{2} \frac{\sigma_{2}}{\sigma_{1}}} .
\end{aligned}
$$

As $I_{a}(\theta)$ for diffractive scattering by large particles is proportional to the fourth power of the radius, if scattering by the collection of droplets were purely incoherent, the intensity that is due to a few large droplets would dominate the intensity that is due to many small ones. This is not observed for a correlated system. Thus the intensity nonadditivity produced by phase correlation effects is expected to be important for this system.

Let $\Delta_{11}\left(\mathbf{r}_{j}-\mathbf{r}_{i}\right)$ be the local microscopic number density of type-1 droplets at $\mathbf{r}_{j}$, given that there is a type-1 droplet at $\mathbf{r}_{i}$, and let $\Delta_{21}\left(\mathbf{r}_{j}-\mathbf{r}_{i}\right)$ be the local microscopic number density of type-2 droplets at $\mathbf{r}_{j}$, given that there is a type- 1 droplet at $\mathbf{r}_{i}$. Let the local microscopic number densities $\Delta_{12}\left(\mathbf{r}_{j}-\mathbf{r}_{i}\right)$ and $\Delta_{22}\left(\mathbf{r}_{j}-\mathbf{r}_{i}\right)$ be defined similarly. There are now four pair-correlation functions that describe fluctuations in the local microscopic number densities from the average macroscopic number densities. ${ }^{17}$ They are defined by

$$
\begin{aligned}
& \Delta_{11}\left(\mathbf{r}_{j}-\mathbf{r}_{i}\right)=g_{11}\left(\mathbf{r}_{j}-\mathbf{r}_{i}\right) \sigma_{1}\left(\mathbf{r}_{j}\right), \\
& \Delta_{21}\left(\mathbf{r}_{j}-\mathbf{r}_{i}\right)=g_{21}\left(\mathbf{r}_{j}-\mathbf{r}_{i}\right) \sigma_{2}\left(\mathbf{r}_{j}\right), \\
& \Delta_{12}\left(\mathbf{r}_{j}-\mathbf{r}_{i}\right)=g_{12}\left(\mathbf{r}_{j}-\mathbf{r}_{i}\right) \sigma_{1}\left(\mathbf{r}_{j}\right), \\
& \Delta_{22}\left(\mathbf{r}_{j}-\mathbf{r}_{i}\right)=g_{22}\left(\mathbf{r}_{j}-\mathbf{r}_{i}\right) \sigma_{2}\left(\mathbf{r}_{j}\right) .
\end{aligned}
$$

For an isotropic system, symmetry dictates that

$$
g_{21}(r)=g_{12}(r) .
$$

The light scattered by this system of particles is

$$
\begin{aligned}
I(\theta)= & N_{1} I_{1}(\theta) S_{11}(\theta)+N_{2} I_{2}(\theta) S_{22}(\theta)+\left(N_{1} N_{2}\right)^{1 / 2} \\
& \times\left[\mathbf{E}_{1}^{*}(\theta) \cdot \mathbf{E}_{2}(\theta)+\mathbf{E}_{2}{ }^{*}(\theta) \cdot \mathbf{E}_{1}(\theta)\right] S_{12}(\theta),
\end{aligned}
$$

where the structure factors $S_{i j}(\theta)$ are given by

$$
\begin{aligned}
S_{i j}(\theta)= & \delta_{i j}+2 \pi\left(\sigma_{i} \sigma_{j}\right)^{1 / 2} \int_{0}^{\infty} r \mathrm{~d} r\left[g_{i j}(r)-1\right] \\
& \times J_{0}(2 k r \sin \theta) .
\end{aligned}
$$

Equation (18) contains two departures from the incoherent additivity of intensities. The first is the type-1-type-1 and type-2-type-2 phase correlation factors of Eq. (19) that were encountered above. The second is the type-1-type-2 phase correlation factor that is proportional to $S_{12}(\theta)$ in Eq. (18).

Analytical expressions for the structure factors for a mixture of $M$ types of noninteracting hard disk are given in Ref. 16. As the number of disk sizes increases, the number of structure factors increases, and their analytical representation becomes more complicated. This is one reason why we consider mixtures of only two types of condensation droplets. The other reason is that the results described in Section 3 are sufficiently general that considering many different sizes would almost certainly lead to the same conclusions. The structure factor expressions in Ref. 16, unfortunately, contain a number of 
misprints. Thus we reproduce the corrected equations here. For disks of a single size we have

$$
S(\theta)=(C(\theta)+1)^{-1},
$$

where, from Ref. 16, Eqs. (4.4), (4.6), (4.8), (4.9), and (6.8),

$$
\begin{aligned}
C(\theta)= & \frac{4 f}{(1-f)}\left[\frac{2 J_{1}(2 k a \sin \theta)}{2 k a \sin \theta}\right] \\
& +\frac{4 f^{2}}{(1-f)^{2}} J_{0}(k a \sin \theta)\left[\frac{2 J_{1}(k a \sin \theta)}{k a \sin \theta}\right] \\
& +\left[\frac{f^{2}}{(1-f)^{2}}+\frac{2 f^{3}}{(1-f)^{3}}\right]\left[\frac{2 J_{1}(k a \sin \theta)}{k a \sin \theta}\right]^{2}
\end{aligned}
$$

This expression satisfies the forward-scattering limit ${ }^{18}$

$$
S(0)=\frac{(1-f)^{3}}{(1+f)}
$$

and exhibits the long-range order-disorder phase transition at $f \approx 0.754$. For a binary system, Ref. 16 , Eq. (4.9), gives the matrix equation

$$
\left[S_{i j}(\theta)\right]=\left[\begin{array}{cc}
C_{11}+1 & C_{12} \\
C_{21} & C_{22}+1
\end{array}\right]^{-1}
$$

where

$$
\begin{aligned}
C_{i i}= & \chi_{2} a_{i}^{2} f_{i}\left[\frac{2 J_{1}\left(k a_{i} \sin \theta\right)}{k a_{i} \sin \theta}\right]^{2}+4 \chi_{1} a_{i} f_{i} J_{0}\left(k a_{i} \sin \theta\right) \\
& \times\left[\frac{2 J_{1}\left(k a_{i} \sin \theta\right)}{k a_{i} \sin \theta}\right]+4 \chi_{0} f_{i}\left[\frac{2 J_{1}\left(2 k a_{i} \sin \theta\right)}{2 k a_{i} \sin \theta}\right], \\
C_{12}= & C_{21}=\chi_{2} a_{1} a_{2}\left(f_{1} f_{2}\right)^{1 / 2}\left[\frac{2 J_{1}\left(k a_{1} \sin \theta\right)}{k a_{1} \sin \theta}\right] \\
& \times\left[\frac{2 J_{1}\left(k a_{2} \sin \theta\right)}{k a_{2} \sin \theta}\right]+2 \chi_{1} a_{2}\left(f_{1} f_{2}\right)^{1 / 2} J_{0}\left(k a_{1} \sin \theta\right) \\
& \times\left[\frac{2 J_{1}\left(k a_{2} \sin \theta\right)}{k a_{2} \sin \theta}\right]+2 \chi_{1} a_{1}\left(f_{1} f_{2}\right)^{1 / 2} J_{0}\left(k a_{2} \sin \theta\right) \\
& \times\left[\frac{2 J_{1}\left(k a_{1} \sin \theta\right)}{k a_{1} \sin \theta}\right]+\chi_{0}\left(f_{1} f_{2}\right)^{1 / 2} \frac{\left(a_{1}+a_{2}\right)^{2}}{a_{1} a_{2}} \\
& \times\left[\frac{2 J_{1}\left(k a_{1} \sin \theta+k a_{2} \sin \theta\right)}{k\left(a_{1}+a_{2}\right) \sin \theta}\right]^{2} \\
\chi_{0}= & (1-f)^{-1}, \\
\chi_{1}= & \left(\frac{f_{1}}{a_{1}}+\frac{f_{2}}{a_{2}}\right)(1-f)^{-2}, \\
\chi_{2}= & \left(\frac{f_{1}}{a_{1}^{2}}+\frac{f_{2}}{a_{2}^{2}}\right)(1-f)^{2}+2\left(\frac{f_{1}}{a_{1}}+\frac{f_{2}}{a_{2}}\right)^{2}(1-f)^{3} .
\end{aligned}
$$

Our model calculations for scattering by condensation droplets on a window pane consist of the use of

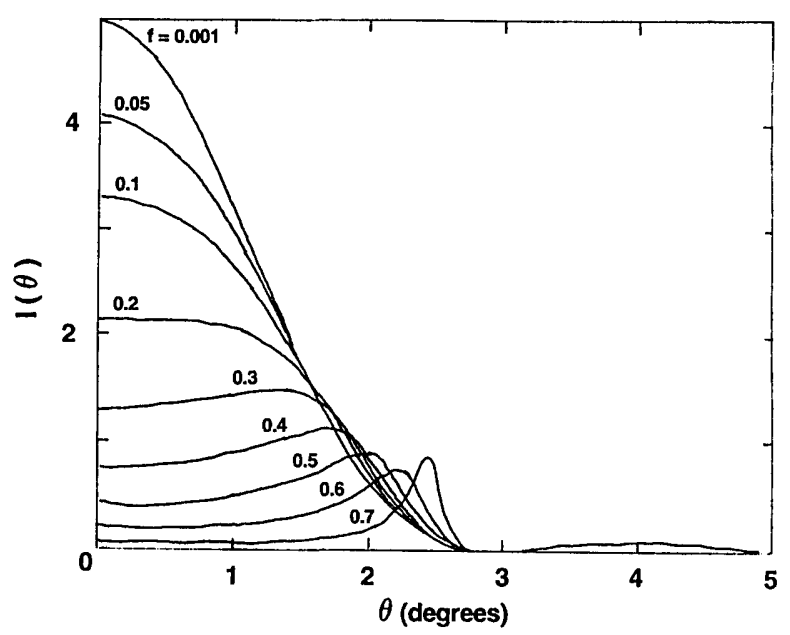

Fig. 3. Scattered intensity as a function of scattering angle from Eqs. (9), (10), (20), and (21) for $a=7.5 \mu \mathrm{m}, \lambda=0.6328 \mu \mathrm{m}$, and for a number of area fractions in the range $0.001 \leq f \leq 0.7$. In this model, the long-range order-disorder phase transition occurs at $f=$ 0.754 .

either Eqs. (9), (10), (20), and (21) for droplets of one size or Eqs. (10), (18), and (23)-(25) for a binary mixture of droplet sizes.

\section{Results of the Model Calculations}

Our model calculations, performed for several monodispersions and binary mixtures of droplets, produced six major results. The first three results are concerned with monodispersions, the next two with binary mixtures of similar size droplets, and the last result is for a binary mixture of vastly dissimilar size droplets. These results are as follows: (1) The scattered intensity was calculated as a function of $\theta$ for a monodispersion with $a=7.5 \mu \mathrm{m}, \lambda=0.6328 \mu \mathrm{m}$ and for many different area fractions in the range $0.001 \leq$ $f \leq 0.7$. The angle $\theta$ ranged from forward scattering to slightly less than the second diffraction minimum at $\theta=5.37^{\circ}$. The results are shown in Fig. 3. For $f \leqslant 0.001$, the droplets are sufficiently dilute that, besides the nonoverlap constraint, there is enough room available so that the droplets have no difficulty in positioning themselves randomly. Thus the correlation function is well approximated by $g(r)=1$ for $r>2 a$, and Eq. (9) reduces to

$$
I(\theta) \approx N I_{a}(\theta) .
$$

The central diffraction maximum appears prominently, corresponding to the situation encountered in cloud corona. ${ }^{4,5}$

(2) When the area fraction is slightly less than that for the order-disorder phase transition, a prominent scattering peak rises up at

$$
\sin \theta \approx \frac{\lambda}{2 a} .
$$

This corresponds to the bright colored ring mentioned in Section 1. This peak grows higher and 
narrower as the area fraction increases and evolves into the coherent first Bragg peak at the orderdisorder transition. ${ }^{7-9}$ Comparison with Eq. (1) shows that for high area fractions, nearest-neighbor droplets are almost touching. Comparison with Eq. (9) shows that this enhancement occurs close to the first diffraction zero. Thus the bright ring of light mentioned in Section 1 is more due to the short-range semiordering of the condensation droplets on the window pane than it is to diffraction by individual droplets. As a result, the bright ring would remain largely unchanged if the condensation droplets did not have exactly circular perimeters and the resulting diffraction pattern of an individual droplet were more complicated. In addition, whenever the dark region is observed inside the bright ring of light, the area fraction of the droplets on the window pane is quite high, at least $f \approx 0.6$ and possibly as high as $f \approx 0.7$.

Figure 4 shows the scattered intensity in greater detail for $f \approx 0.7$ just below the long-range orderdisorder transition. In addition to the near-forward angle scattering suppression and the first scattering peak of expression (27), there is a much weaker and broader second scattering peak at

$$
\sin \theta \approx \frac{1.84 \lambda}{2 a}
$$

If this were due solely to diffraction, it would occur at $\sin \theta \approx 1.72 \lambda / 2 a$, and if it were due solely to short-range ordering, it would occur at $\sin \theta \approx$ $2 \lambda / 2 a$. The actual peak represents a combination of both effects.

(3) Plate 1 shows correlated scattering that is due to haze on a camera lens focused on a distant mercury street lamp. The light from the street lamp, although appearing bluish white to the naked eye, is in actuality dominated by four spectral lines, blue at $\lambda_{B}=0.4358 \mu \mathrm{m}$, green at $\lambda_{G}=0.5461 \mu \mathrm{m}$, yellow at $\lambda_{\mathrm{Y}}=0.5791 \mu \mathrm{m}$, and red at $\lambda_{\mathrm{R}}=0.6198 \mu \mathrm{m}$. The

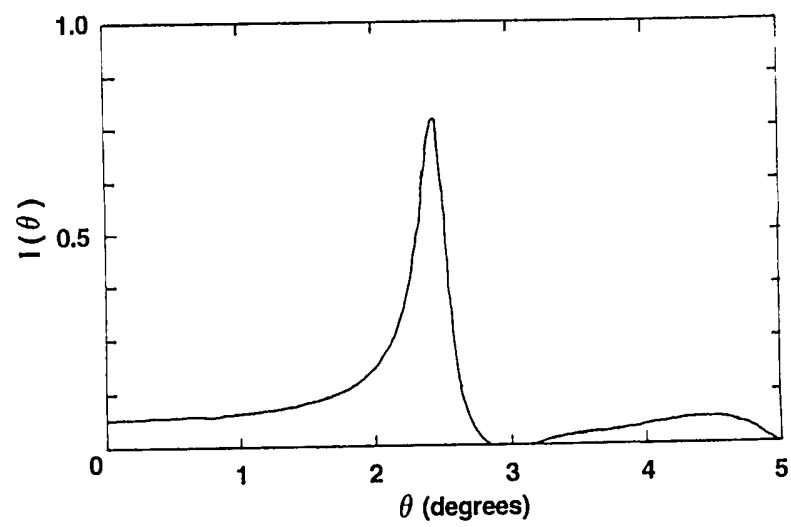

Fig. 4. Scattered intensity as a function of scattering angle from Eqs. (9), (10), (20), and (21) for $a=7.5 \mu \mathrm{m}, \lambda=0.6328 \mu \mathrm{m}$, and $f=$ 0.7 . The first scattering peak occurs at $\sin \theta \approx \lambda / 2 a$, and the sccond scattering peak occurs at $\sin \theta \approx 1.84 \lambda / 2 a$.

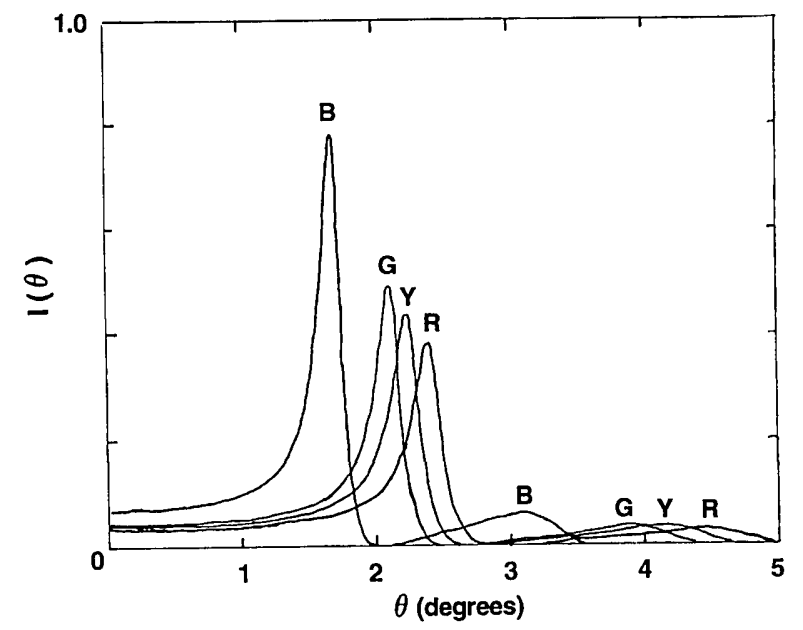

Fig. 5. Scattered intensity as a function of scattering angle from Eqs. (9), (10), (20), and (21) for $a=7.5 \mu \mathrm{m}, \lambda=0.4358 \mu \mathrm{m}$ (B), $\lambda=$ $0.5461 \mu \mathrm{m}(\mathrm{G}), \lambda=0.5791 \mu \mathrm{m}(\mathrm{Y})$, and $\lambda=0.6198 \mu \mathrm{m}(\mathrm{R})$, and for $f=0.7$.

blue, green, and yellow lines are produced in the emission spectrum of mercury, and the red line is produced by fluorescence of a coating on the inner surface of the glass envelope of the lamp. ${ }^{19}$ Figure 5 shows scattering that is due to the mercury street lamp wavelengths. The relative intensities of $\lambda_{B}, \lambda_{G}$, $\lambda_{Y}$, and $\lambda_{R}$ and the relative sensitivity of the human visual system to these wavelengths have not been taken into account. Still, the narrowness of the first scattering peak for $f \approx 0.7$ implies that good color resolution should occur at high-area fractions. Although Figs. 3-5 were calculated for $a=7.5 \mu \mathrm{m}$, the results are identical for any other wavelength or droplet size, except for a scaling factor, as each term in Eqs. (9), (10), (20), and (21) is a function of only $k a$ $\sin \theta$.

(4) Consider a clean glass surface that is relatively free of fingerprints, grease spots, and dirt. In the absence of these large potential nucleation sites, moisture condenses at all locations on the window pane at roughly the same rate, producing nearly equal-size condensation droplets. To model this situation we considered binary mixtures of droplets in the range $1.0<a_{2} / a_{1} \leq 1.4$. Typical results for equal numbers of the two types of droplet and for $f=0.7$ are shown in Fig. 6. If the scattering were purely incoherent, the angular width of the first scattering peak would extend from the inner edge of the $a_{2}$ peak to the outer edge of the $a_{1}$ peak. The nonadditivity produced by phase correlation narrows the binary mixture scattering peak to being no wider than either of the two component droplet peaks. Thus, if the two types of droplet are similar in size, their individual light-scattering signatures are not observed. Rather, they act as a single semiordered structure of monodisperse particles of some average radius. This radius was found to be well approximated by the intensity- 


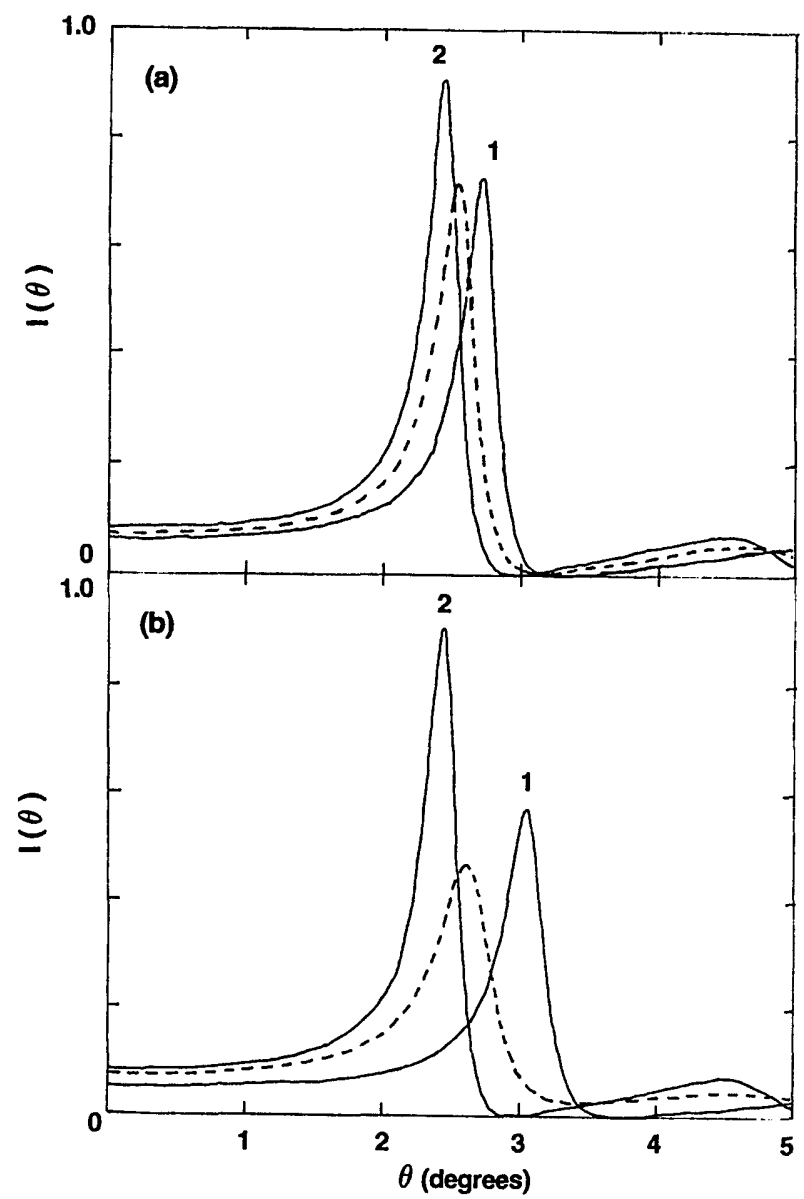

Fig. 6. (a) Scattered intensity as a function of scattering angle for $a_{1}=6.75 \mu \mathrm{m}, f_{1}=0.7$ (curve 1); $a_{2}=7.5 \mu \mathrm{m}, f_{2}=0.7$ (curve 2); and the binary mixture $a_{1}=6.75 \mu \mathrm{m}, a_{2}=7.5 \mu \mathrm{m}, f_{1}=0.3133, f_{2}=$ 0.3867 , and $\sigma_{1}=\sigma_{2}$ (dashed curve). All these curves are for $\lambda=$ $0.6328 \mu \mathrm{m}$. The size ratio is $a_{2} / a_{1}=1.11$. (b) Scattered intensity as a function of scattering angle for $a_{1}=6.0 \mu \mathrm{m}, f_{1}=0.7$ (curve 1); $a_{2}=7.5 \mu \mathrm{m}, f_{2}=0.7$ (curve 2); and the binary mixture $a_{1}=6.0$ $\mu \mathrm{m}, a_{2}=7.5 \mu \mathrm{m}, f_{1}=0.2732, f_{2}=0.4268$, and $\sigma_{1}=\sigma_{2}$ (dashed curve). All these curves are for $\lambda=00.6328 \mu \mathrm{m}$. The size ratio is $a_{2} / a_{1}=1.25$.

weighted average

$$
a_{\text {ave }}=\frac{\left(a_{1}^{4} \sigma_{1}\right) a_{1}+\left(a_{2}{ }^{4} \sigma_{2}\right) a_{2}}{a_{1}{ }^{4} \sigma_{1}+a_{2}{ }^{4} \sigma_{2}},
$$

producing a first scattering peak at

$$
\sin \theta \approx \frac{\lambda}{2 a_{\mathrm{ave}}} .
$$

(5) The continued narrowness of the first scattering peak for high-area fractions and a reasonably narrow polydispersion of droplet sizes yield good color resolution of the light from a distant mercury street lamp. This is shown in Fig. 7. Again the relative strength of the four street lamp wavelengths and the relative sensitivity of the human visual system have not been taken into account. But the important point is that

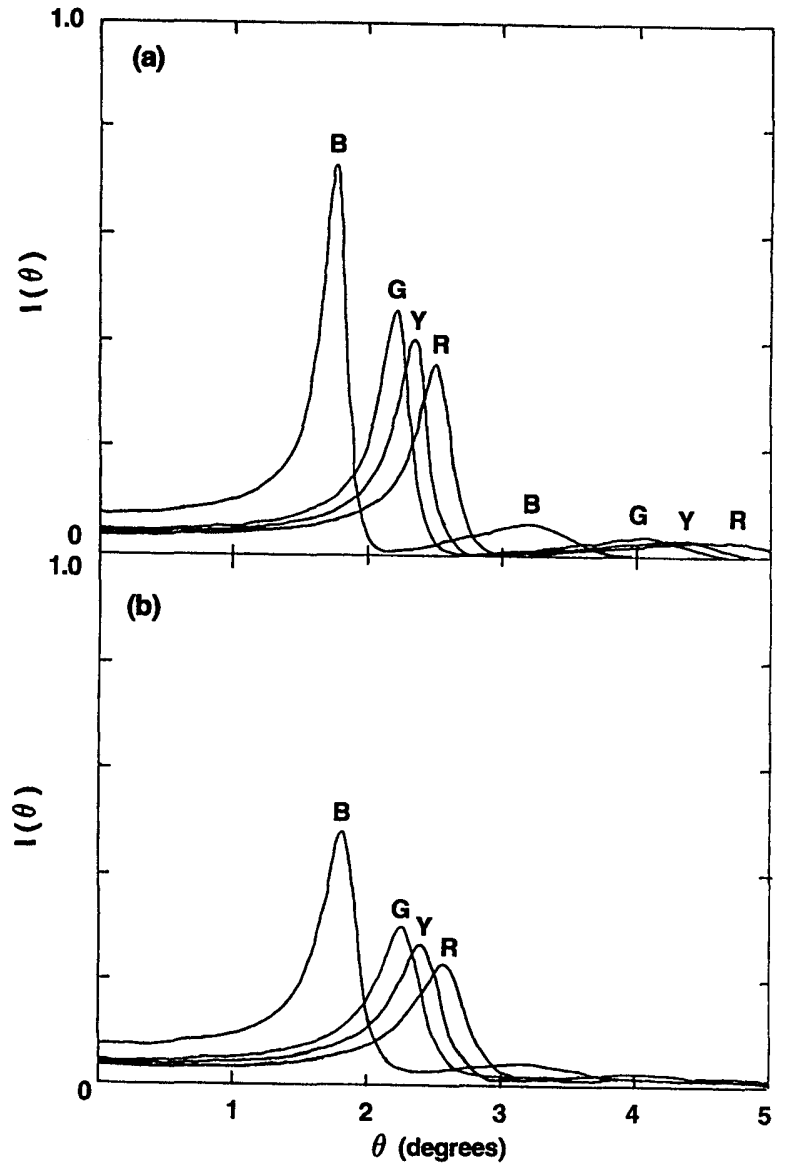

Fig. 7. (a) Scattered intensity as a function of scattering angle for the binary mixture of Fig. $6(\mathrm{a})$ and $\lambda=0.4358 \mu \mathrm{m}(\mathrm{B}), \lambda=0.5461$ $\mu \mathrm{m}(\mathrm{G}), \lambda=0.5891 \mu \mathrm{m}(\mathrm{Y})$, and $\lambda=0.6198 \mu \mathrm{m}(\mathrm{R})$. (b) Scattered intensity as a function of scattering angle for the binary mixture of Fig. 6(b) and the wavelengths of Fig. 7(a).

the condensation droplets do not have to be monodisperse for a bright colored ring of light to be observed through a fogged window pane. A reasonably narrow polydispersion of droplet sizes produces the effect just as well, leading to the frequency of this observation. This situation is contrasted with cloud corona, where it is uncommon to see more than the aureole ring, as the polydispersion of cloud droplets must be much narrower to see additional rings. ${ }^{5}$

(6) Now consider a glass surface that has not been precleaned. Small particles of dirt and grease on the surface act as large nucleation sites for condensation. As a result both small condensation droplets as above and more substantial droplets that are due to the larger nucleation sites form. We modeled this situation with binary mixtures of droplets that have $2.0 \leq$ $a_{2} / a_{1} \leq$ 3.0. Sample results are shown in Fig. 8 . For $a_{2} / a_{1}=2.0$ and purely incoherent scattering, each type-2 droplet should diffractively scatter $2^{4}=$ 16 times more light than each type- 1 droplet. As there are 2.57 more type-1 droplets than type-2 droplets, the type-2 scattering peak should be 6.2 times as intense as the type-1 scattering peak. In actuality, for the binary mixture, phase correlation 


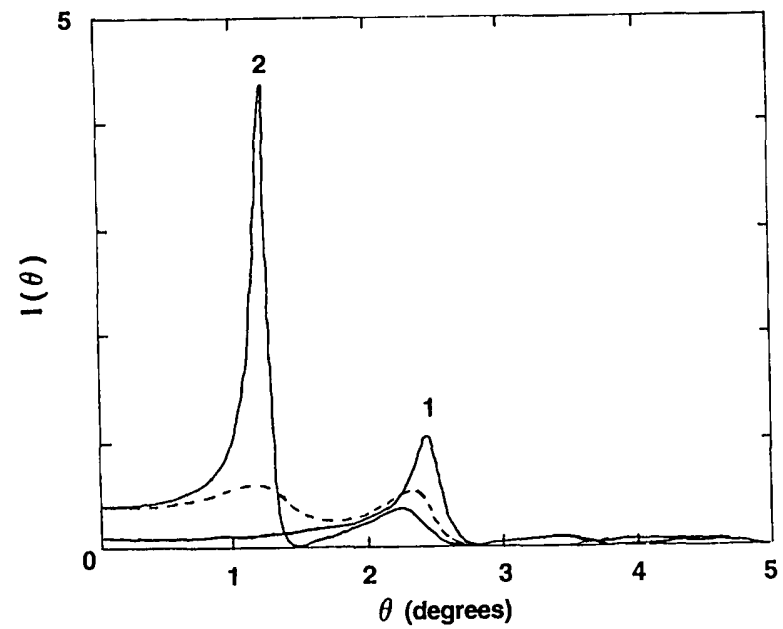

Fig. 8. Scattered intensity as a function of scattering angle for $a_{1}=7.5 \mu \mathrm{m}, f_{1}=0.7$ (curve 1); $a_{2}=15.0 \mu \mathrm{m}, f_{2}=0.7$ (curve) 2 ); and the binary mixture $a_{1}=7.5 \mu \mathrm{m}, a_{2}=15.0 \mu \mathrm{m}, f_{1}=0.275, f_{2}=$ 0.425 , and $\sigma_{2}=2.57 \sigma_{1}$ (dashed curve). All the curves are for $\lambda=$ $0.6328 \mu \mathrm{m}$. The size ratio is $a_{2} / a_{1}=2.0$.

renders both peaks equally intense. Also significant is the fact that the size difference between the two droplet types is now large enough that both component peaks appear separately at

$$
\begin{aligned}
& \sin \theta_{1} \approx \frac{\lambda}{2 a_{1}}, \\
& \sin \theta_{2} \approx \frac{\lambda}{2 a_{2}} .
\end{aligned}
$$

The mixture acts as two interpenetrating semiordered structures, one of nearest-neighbor separation $2 a_{1}$ and the other of nearest-neighbor separation $2 a_{2}$. Furthermore, if many droplet sizes were present and the range in sizes were a factor of 2 or larger, the scattered intensity would no longer possess the nearforward direction suppression. The intensity would be a rather featureless monatonically decreasing function of $\theta$.

\section{Photographic Analysis}

A number of photographs were taken of a mercury street lamp $\sim 45 \mathrm{~m}$ away. We determined the wavelengths of the lamp's four component colors by photographing the lamp through a diffraction grating and by checking the results with the published mercury emission spectrum. ${ }^{20}$ Before each condensation droplet photograph was taken, the camera lens was cleaned. It was then breathed on in order to produce the condensation layer on the lens. The condensation layer evaporated in $\sim 5 \mathrm{~s}$. During the evaporation, the bright ring of light that is due to the first scattering peak remained unaffected, indicating that evaporation proceeded by the droplets' thinning while preserving the same area of contact on the window pane. ${ }^{1}$ A representative photograph of the bright scattering ring and the near-forward angle scattering suppression is shown in Plate $44 .^{21}$ In each condensation droplet photograph the angular size of the red edge $\left(\lambda_{R}=0.6198 \mu \mathrm{m}\right)$ and the blue edge $\left(\lambda_{B}=0.4358 \mu \mathrm{m}\right)$ of the bright ring of light was determined by scaling from the angular size of the image of the street lamp. Using expression (30), we obtained the average radius of the droplet size distribution. These results are shown in Table 1. The convolution of the scattering pattern with the angular size of the street lamp was taken into account. The droplet radii we obtained by using the blue and red edges of the bright scattering ring are similar, indicating the internal consistency of expression (30). It is also interesting that the size of the condensation droplets is generally reproducible, there being only a $50 \%$ difference between the largest and the smallest values of $a_{\text {ave }}$ obtained in different trials.

A number of condensation droplet photographs were also taken when the camera lens was not cleaned between exposures. Residue from evaporation gradually built up on the lens, providing large nucleation sites, and within a few trials, scattering by the haze on the lens resembled the situation shown in Plate 45. Here the size distribution of condensation droplets is bimodal, with the smaller scattering ring corresponding to $a_{\text {ave }} \approx 37 \mu \mathrm{m}$ and the larger scattering ring corresponding to $a_{\text {ave }} \approx 11 \mu \mathrm{m}$. The situation resembles the calculated intensity of Fig. 8. When the lens had not been cleaned, a few exposures later the intensity profile became rather featureless, as in Plate 46 , which indicates a wide polydispersion of condensation droplet sizes.

As a final consistency check, the pair-correlation function model of correlated scattering assumes a coherent light source so that the phases of the light scattered by the different droplets are correlated in the proper manner. The mercury street lamp, however, had an angular size of $0.24^{\circ}$, as seen by the observer $45 \mathrm{~m}$ away. The resulting coherence width of the mercury source at the window pane is then ${ }^{22}$

$$
W \approx \frac{0.32 \lambda}{\Delta \theta}=50 \mu \mathrm{m} .
$$

A given condensation droplet and its nearest neighbors lie within the source coherence width, and as a

$$
\begin{aligned}
& \text { Table 1. Average Droplet Radius from Expression }(30) \text { based on the } \\
& \text { Blue Edge }(0.4358 \mu \mathrm{m}) \text { and the Red Edge }(0.6198 \mu \mathrm{m}) \text { of the Bright } \\
& \text { Scattering Ring for Eight Photographs of Correlated Scattering } \\
& \text { Produced by Condensation Droplets on a Camera Lens }{ }^{a}
\end{aligned}
$$

\begin{tabular}{ccc}
\hline $\begin{array}{c}\text { Photograph } \\
\text { Number }\end{array}$ & $a_{\text {ave }}{ }^{0.4358 \mu \mathrm{m}}(\mu \mathrm{m})$ & $a_{\text {ave }}{ }^{0.6198 \mu \mathrm{m}}(\mu \mathrm{m})$ \\
\hline 4 & 17.4 & 15.7 \\
8 & 14.5 & 16.1 \\
10 & 12.8 & 16.4 \\
18 & 16.4 & 17.2 \\
19 & 12.0 & 12.0 \\
21 & 12.2 & 11.2 \\
22 & 12.8 & 13.4 \\
23 & 15.3 & 12.4 \\
\hline
\end{tabular}

${ }^{a}$ All average radii are $\pm 0.6 \mu \mathrm{m}$. 
result, the phases of their scattered light are correlated as described in Section 2. In summary, although the two-size model of correlated scattering is successful in predicting where the bright ring of scattered light occurs, the pair-correlation-function model provides valuable physical insight as to how the scattered intensity behaves when condensation droplets of differing sizes are present on the window pane. It also predicts the densities that are required for producing the near-forward direction scattering suppression.

This work was supported in part by the National Aeronautics and Space Administration grant \#NCC 3-204.

\section{References and Notes}

1. M. Minnaert, The Nature of Light and Color in the Open Air (Dover, New York, 1954), article 162.

2. J. J. M. Reesinck and D. A. de Vries, "The diffraction of light by a large number of circular objects," Physica 7, 603-608 (1940).

3. J. A. Prins, "Scattering by an assembly of molecules," in Electromagnetic Scattering, R. L. Rowell and R. S. Stein, eds. (Gordon \& Breach, New York, 1967), pp. 295-303.

4. R. A. R. Tricker, Introduction to Meteorological Optics (American Elsevier, New York, 1970), Chap. 5.

5. J. A. Lock and L. Yang, "Mie theory model of the corona," Appl. Opt. 30, 3408-3414 (1991).

6. The difference between correlated scattering and other types of scattering is stated especially clearly in the Introduction of C. Smart, R. Jacobsen, M. Kerker, J. P. Kratohvil, and E. Matijevic, "Experimental study of mulitple light scattering," J. Opt. Soc. Am. 55, 947-955 (1965).

7. T. Alfrey, E. B. Bradford, J. W. Vanderhoff, and G. Oster, "Optical properties of uniform particle-size latexes," J. Opt. Soc. Am. 44, 603-609 (1954).

8. I. M. Krieger and F. M. O'Neill, "Diffraction of light by arrays of colloidal spheres,” J. Am. Chem. Soc. 90, 3114-3120 (1968).
9. J. W. Goodwin, R. H. Ottewill, and A. Parentich, "Optical examination of structured colloidal dispersions," J. Phys. Chem. 84, 1580-1586 (1980).

10. E. D. Freund, R. L. McCally, and R. A. Farrell, "Direct summation of fields for light scattering by fibrils with applications to normal corneas," Appl. Opt. 25, 2739-2746 (1986).

11. R. W. Hart and R. A. Farrell, "Light scattering in the cornea," J. Opt. Soc. Am. 59, 766-774 (1969).

12. W. W. Wood, "Monte Carlo studies of simple liquid models," in The Physics of Simple Liquids, H. N. V. Temperley, J. S. Rowlinson, and G. S. Rushbrooke, eds. (North-Holland, Amsterdam, 1968), Chap. 5.

13. D. G. Chae, F. H. Ree, and T. Ree, "Radial distribution functions and equation of state of the hard-disk fluid," $J$. Chem. Phys. 50, 1581-1589 (1969).

14. W. W. Wood, "NpT-ensemble Monte Carlo calculations for the hard-disk fluid," J. Chem. Phys. 52, 729-741 (1970).

15. Y. Uehara, T. Ree, and F. H. Ree, "Radial distribution function for hard disks from the BGY2 theory," J. Chem. Phys. 70, 1876-1883 (1979).

16. Y. Rosenfeld, "Free-energy model for the inhomogeneous hard-sphere fluid in $D$ dimensions: Structure factors for the hard disk $(D=2)$ mixtures in simple explicit form," Phys. Rev. A 42, 5978-5989 (1990).

17. J.-L. Barrat, H. Xu, J.-P. Hansen, and M. Baus, "Freezing of binary hard-disc alloys: I. The equation of state and pair structure of the fluid phase," J. Phys. C 21, 3165-3176 (1988).

18. V. Twersky, "Transparency of pair-correlated, random distributions of small scatterers, with applications to the cornea," J. Opt. Soc. Am. 65, 524-530 (1975), Eq. (22).

19. General Electric Lamp Specification Bull. LSB\#206-0155R1 (General Electric, Cleveland, Ohio, 1980).

20. D. E. Gray, ed., American Institute of Physics Handbook (McGraw-Hill, New York, 1963), pp. 7.122-7.125.

21. See also Sky and Telescope, December 1993, p. 52.

22. E. Hecht, Optics, 2nd ed. (Addison-Wesley, Reading, Mass., 1987), p. 532. 